\title{
Structural, functional, and lectin histochemical characteristics of rat ovaries and endometrium in experimental hyper- and hypothyroidism
}

\author{
Alexander Lutsyk, Elisabeth Sogomonian \\ Department of Histology and Embryology, Lviv National Medical University, Lviv, Ukraine
}

\begin{abstract}
Twenty lectins with different carbohydrate affinities, including five new lectins purified from fungi, were used for histochemical examination of carbohydrate determinants in rat ovaries and endometrium under experimental hyper- and hypothyroidism. In the ovaries of control rats, lectin binding was detected predominantly in the zona pellucida and corona radiata of growing follicles, luteocytes, and leucocytes. Within the endometrium, lectins reacted strongly with the luminal surface, epitheliocytes and secretions of the uterine glands, pre-decidual cells, leucocytes, and collagen fibers. As revealed on general morphology and estrous cycle studies, hyperthyroidism induced ovarian luteinization, and increased the content of pre-decidual cells and of collagen fibers in the endometrium, with no reliable effect on the estrous cycle. Hypothyroidism was accompanied by retardation of the estrous cycle, enhanced folliculogenesis and leucocyte infiltration of ovarian and endometrial stroma. Both hyper- and hypothyroidism significantly affected the tissue carbohydrates of the ovaries and endometrium: specific and differential redistribution of DMan, LFuc, NeuNAc, DGlcNAc, DGalNAc, and DGal determinants was detected under both pathological conditions. Hyperthyroidism induced more severe alterations of glycoconjugates than hypothyroidism; the endometrium was more susceptible to thyroxin-modulated impairments than the ovaries. These results give new insights into the pathogenetic mechanisms of the effects of thyroid disorders on the female reproductive organs and demonstrate the suitability of lectin histochemistry methods for monitoring the efficacy of hormonal imbalance correction therapy, as well as the applicability of new lectin preparations for the selective labeling of ovarian and endometrial constituents. (Folia Histochemica et Cytobiologica 2012, Vol. 50, No. 3, 331-339)
\end{abstract}

Key words: lectin histochemistry, rat ovaries, rat endometrium, experimental hyperthyroidism, experimental hypothyroidism

\section{Introduction}

Thyroid disorders are currently among the most widespread endocrine pathologies, affecting about $3 \%$ of the world's population [1]. In Ukraine over the past ten years, the incidence of thyroid disorders, predominantly due to Chornobyl accident contaminants, has increased three-fold [2]. In addition, the population of

Correspondence address: A.D. Lutsyk,

Department of Histology and Embryology,

Lviv National Medical University,

Pekarska Str. 69, 79010 Lviv, Ukraine;

tel./fax: + 3803227559 47;

e-mail: lutsyk@meduniv.lviv.ua the Carpathian region, living in an area where iodine deficiency is endemic, requires special care [3].

The thyroid gland plays an important role in the histophysiology of the female reproductive system. Both hyperthyroidism and hypothyroidism may affect sexual maturation and menstrual function, inhibit fertility, and increase the rates of gynecological disorders, spontaneous abortions, and fetal mortality $[4-8,20]$. A literature survey revealed that, despite numerous clinical observations correlating thyroid pathologies and reproductive dysfunction, the theoretical aspects of this problem are far from being completely resolved.

The crucial roles of carbohydrate determinants during oocyte maturation, fertilization, implantation, gastrulation, and cell sorting, have been well docu- 
mented [9-13]. Therefore, glycoconjugate rearrangement in the female reproductive organs under different pathological conditions can be of special interest for clinical and experimental medicine. The relevant literature lacks information reflecting the effect of thyroid hormone imbalance on the glycome of the female reproductive organs.

The aim of this study was to use a panel of lectins with different carbohydrate affinities to investigate carbohydrate determinant redistribution in rat ovaries and endometrium under experimental hyper- and hypothyroidism.

\section{Material and methods}

Animals. The study was performed on 65 female Wistar rats, subdivided into five groups: control, hyperthyroid, hypothyroid, and two groups subjected to simultaneous induction of hypothyroidism and thyroxin therapy. Hyperthyroidism was induced with $100 \mu \mathrm{g} / \mathrm{kg}$ of L-thyroxin (Pharmak, Kyiv, Ukraine), and hypothyroidism was induced with $10 \mathrm{mg} / \mathrm{kg}$ of the antithyroid drug mercazolil (1-methyl-2-mercaptoimidazole, Zdorovja, Kharkiv, Ukraine), which were added daily in powdered form to the rats' food allowance for 14 days. Replacement therapy was maintained by simultaneous food supplementation of mercazolil-treated animals with thyroxin: first replacement group $-1.6 \mu \mathrm{g} / \mathrm{kg}$; and the second replacement group $-30 \mu \mathrm{g} / \mathrm{kg}$. Starting from day 15 of the experiment, simultaneously with thyroxin or mercazolil treatment, microscopic investigation of vaginal smears (estrous cycle analysis) was performed for the following 21 days.

The investigation was carried out according to the ethical criteria for the use and handling of laboratory animals established by Lviv National Medical University in accordance with the 'General ethical principles on experiments with animals' of the $1^{\text {st }}$ National Congress on Bioethics (Kiev, 2001) and in compliance with the National Institutes of Health (NIH) Guide for the Care and Use of Laboratory Animals (1996).

Tissue specimens. Rats were sacrificed during the diestral period of the cycle by diethyl narcosis overdose. The ovaries, uterine wall (midpiece of the uterine horns), and thyroid glands were excised, fixed in $4 \%$ neutral formalin, and embedded in paraffin. For general morphology, 5- to 7- $\mu \mathrm{m}$ thick tissue sections were stained with hematoxylin-eosin. Thyroid specimens were examined to morphologically confirm the induction of hyper- or hypothyroidism. In particular, thyroxin overdose was accompanied by significantly lowered epithelium and compacted colloid within the thyroid follicles, while, on the other hand, mercazolil treatment was associated with increased activity of thyroid follicles, including enhanced thyrocyte height and colloid resorption.

Lectin histochemistry. The lectin panel included 15 conventional lectins, supplemented with five new lectins puri- fied from fungi [14] (Table 1). All lectins and their peroxidase conjugates were prepared by Dr. V. Antonyuk (Lectinotest, Lviv, Ukraine). Carbohydrate determinants were visualized according to the lectin-peroxidase-diaminobenzidine staining protocol [15].

In detail, deparaffinized sections were incubated for $20 \mathrm{~min}$ in methanol containing $0.3 \% \mathrm{H}_{2} \mathrm{O}_{2}$ to block activity of endogenous peroxidase, through graded ethanol brought to phosphate buffered saline (PBS) $\mathrm{pH} 7.4$, rinsed in three portions of PBS (5 min each), and incubated for 45 min with lectin-peroxidase conjugates (dilution 25-50 $\mu \mathrm{g} / \mathrm{ml}$ in PBS) in a moist chamber at room temperature. Lectin receptor sites were visualized in PBS, containing $0.05 \%$ diaminobenzidine (Sigma, St. Louis, MO, USA) and $0.015 \% \mathrm{H}_{2} \mathrm{O}_{2}$. Thereafter slides were twice washed in distilled water, and after dehydration mounted in balsam.

The specificity of histochemical reactions was controlled by: (1) omitting lectin-peroxidase from the staining protocol; and (2) pre-incubation of tissue sections prior to lectin labeling for $60 \mathrm{~min}$ in $1 \% \mathrm{HIO}_{4}$ (Reanal, Budapest, Hungary) for oxidative damage of carbohydrate determinants. In both cases, staining results were negative.

Microscopic investigation was performed using a Carl Zeiss Ng (Jena, Germany) microscope equipped with a Canon IXUS 700 digital camera (Canon, Tokyo, Japan). For the evaluation of lectin binding, two investigators performed the analysis independently, blinded to lectin type. Binding intensity was represented in the semiquantitative scale as follows: ++ strong, + positive, - negative labeling.

\section{Results}

\section{Estrous cycle}

Experimental hyperthyroidism had no reliable effect on the frequency and duration of the estrous cycle. On the other hand, hypothyroidism induced retardation of the estrous cycle: over 21 days of observation, there were $4.7 \pm 0.24$ estruses in the control group compared to $3.5 \pm 0.24$ estruses in the mercazolil-treated group. The duration of estruses decreased significantly from $10.7 \pm 0.62$ days in the control group to $5.9 \pm 1.5$ days in the hypothyroid group. Similar retardation of estrous cycles was also detected in rats that received mercazolil in combination with $1.6 \mu \mathrm{g} / \mathrm{kg}$ of thyroxin, while animals given combined mercazolil and $30 \mu \mathrm{g} / \mathrm{kg}$ of thyroxin demonstrated estrous cycle parameters similar to those of control rats. These results indicate that estrous cycle retardation was induced by antithyroid activity, not as a side effect of mercazolil.

\section{General morphology}

The ovaries of hyperthyroid rats characteristically showed an enlarged number of hypertrophic corpora 
Table 1. Lectins used and their carbohydrate specificity

\begin{tabular}{|c|c|c|c|}
\hline $\mathbf{N}^{\circ}$ & $\begin{array}{l}\text { Lectin designation, } \\
\text { abbreviation }\end{array}$ & $\begin{array}{c}\text { Specific } \\
\text { monosaccharide }\end{array}$ & $\begin{array}{c}\text { Complementary oligosaccharide/ } \\
\text { /polysaccharide* }\end{array}$ \\
\hline 1. & Canavalia ensiformis agglutinin, Con A & $\alpha \mathrm{DMan} / \alpha \mathrm{DGlc}$ & Glycogen \\
\hline 2. & Lens culinaris agglutinin, LCA & $\alpha$ DMan $/ \alpha$ DGlc & \\
\hline 3. & Narcissus pseudonarcissus agglutinin, NPA & $\alpha$ DMan & $\begin{array}{c}\operatorname{Man}(\alpha 1-3 / 6) \operatorname{Man} \\
\operatorname{Man}(\alpha 1-3) \operatorname{Man}(\beta 1-4) \text { GlcNAc }\end{array}$ \\
\hline 4. & Perca fluviatilis agglutinin, PFA & $\alpha$ LFuc & $\operatorname{Fuc}(\alpha 1-2) \mathrm{DGal}(\beta 1-3) \mathrm{DGalNAc}$ \\
\hline 5. & Lucioperca lucioperca agglutinin, LLA & $\alpha$ LFuc & \\
\hline 6. & Laburnum anagyroides bark agglutinin, LABA & LFuc & $\operatorname{Gal}(\beta 1-4) \operatorname{Fuc}(\beta 1-3) \mathrm{Glc}$ \\
\hline 7. & Laburnum anagyroides seed agglutinin, LASA & LFuc, DGal & \\
\hline 8. & Evonymus europaeus agglutinin, EEA & LFuc, DGlcNAc & $\operatorname{LFuc}(\alpha 1-2) \mathrm{DGal}(\beta 1-3) \mathrm{DGal}$ \\
\hline 9. & Sambucus nigra agglutinin, SNA & $\operatorname{NeuNAc}(\alpha 2-6) \mathrm{DGal}$ & $\operatorname{NeuNAc}(\alpha 2-6) \operatorname{Gal}(\beta 1-4) \operatorname{GlcNAc}(\beta 1-2)$ \\
\hline 10. & Ricinus communis agglutinin, $\mathrm{RCA}$ & $\beta$ DGal $>$ NeuNAc & NeuNAc(2-6)Gal $(\beta 1-4)$ GlcNAc \\
\hline 11. & Wheat germ agglutinin, WGA & DGlcNAc $>$ NeuNAc & $\begin{array}{l}\text { NeuNAc }(\alpha 2-6) \operatorname{Gal}(\beta 1-4) \text { GlcNAc, } \\
\operatorname{Man}(\beta 1-4) \text { GlcNAc }(\beta 1-4) \text { GlcNAc }\end{array}$ \\
\hline 12. & Urtica dioica agglutinin, UDA & DGlcNAc & $(\text { DGlcNAc })_{3}$ \\
\hline 13. & Peanut agglutinin, $\mathrm{PNA}$ & DGal & $\operatorname{DGal}(\beta 1-3)$ GalNAc \\
\hline 14. & Soybean agglutinin, $\mathrm{SBA}$ & $\alpha$ DGalNAc $>\beta$ DGalNAc & $\operatorname{GalNAc}(\alpha 1-3) \operatorname{Gal}(\beta 1-3)$ GalNAc \\
\hline 15. & Helix pomatia agglutinin, HPA & $\alpha$ DGalNAc & $\operatorname{GalNAc}(\alpha 1-3)$ GalNAc \\
\hline 16. & Mycena pura fungus agglutinin, MPFA & $\alpha$ DMan, DGlcNAc & $\operatorname{GlcNAc}(\beta 1-2) \operatorname{Man}(\alpha 1-6) /(\alpha 1-2)$ \\
\hline 17. & Lactarius pergamentus fungus agglutinin, LPFA & DGal & $\begin{array}{l}\mathrm{DGal}(\beta 1-3) \mathrm{DGlcNAc}, \\
\text { complex oligosaccharides }\end{array}$ \\
\hline 18. & Lactarius torminosus fungus agglutinin, LTFA & DGal & $\begin{array}{l}\text { DGal }(\beta 1-3) \text { DGlcNAc, } \\
\text { complex oligosaccharides }\end{array}$ \\
\hline 19. & Paxillus atrotomentus fungus agglutinin, PAFA & DGal & Complex oligosaccharides \\
\hline 20. & Laetiporus sulphureus fungus agglutinin, LSFA & DGal & $\begin{array}{c}\operatorname{DGal}(\beta 1-4) \mathrm{DGlcNAc} \\
\operatorname{DGal}(\beta 1-4) \operatorname{DGlcNAc}(1-3) \mathrm{Gal}(1-4) \mathrm{Glc}\end{array}$ \\
\hline
\end{tabular}

* More detailed information on carbohydrate affinities of used lectins is presented in [14, 15]

lutea, while hypothyroidism induced increased counts of growing and atretic follicles and decreased counts of corpora lutea in association with leucocyte infiltration of the ovarian medulla.

The endometrium in hyperthyroidism showed distension of the vascular bed and increased amounts of pre-decidual cells and collagen fibers. Hypothyroidism was accompanied by heavy leucocyte infiltration of the endometrial stroma.

\section{Lectin histochemistry}

In the ovaries of control rats, all lectins reacted with the zona pellucida and corona radiata, cytoplasmic granularity of oocytes in growing follicles, and luteocytes of corpora lutea (Table 2). Several lectins (NPA, PNA, MPFA, LTFA) expressed high affinity for follicular fluid. The most selective affinity for the zona pellucida and the corona radiata of different types of follicles, as well as for corpora lutea demonstrated
SBA (Figure 1A). Moreover, WGA and UDA intensely labeled the hyalinized zona pellucida of atretic follicles.

Within the endometrium, the lectin label was attached primarily to its luminal surface, apical compartments of glandular epitheliocytes, secretions of some glands, and cytoplasmic glycoconjugates of pre-decidual cells, leucocytes, and collagen fibers (Table 3 ). The best selectivity of leucocyte labeling was obtained with WGA, PNA, UDA, SBA, and HPA. The collagen fibers in the endometrium of control rats expressed most selective affinity for SNA and SBA (Figure 2A).

Of special interest was the histochemical reactivity of new lectin preparations: MPFA selectively labeled the zona pellucida in atretic follicles. PAFA expressed preferential reactivity for the zona pellucida and corona radiata in growing follicles. LPFA, LTFA, LSFA, and PAFA, all specific to DGal residues of complex carbohydrates, in fact expressed differential affinity to ovarian and endometrial components. 


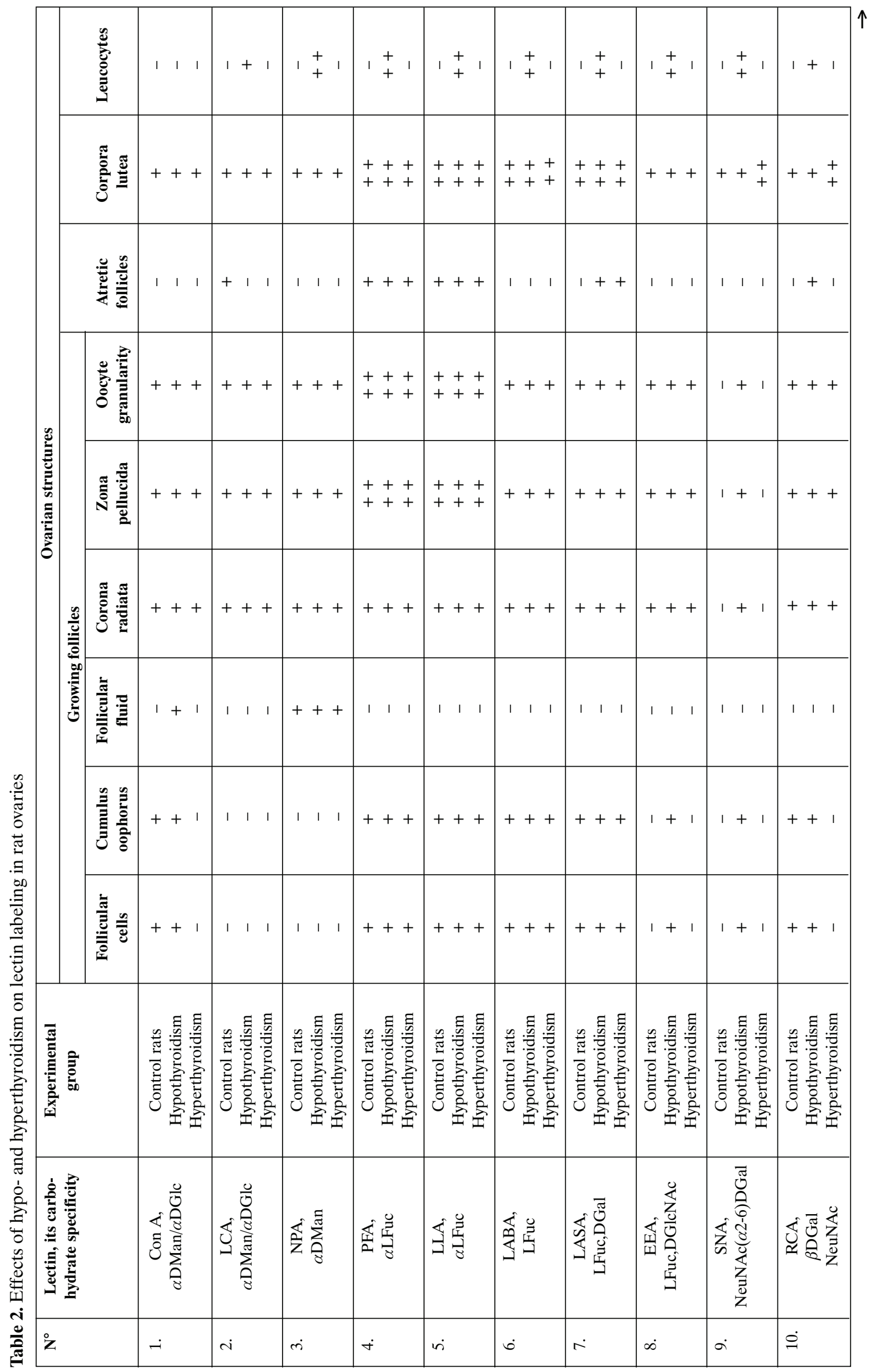




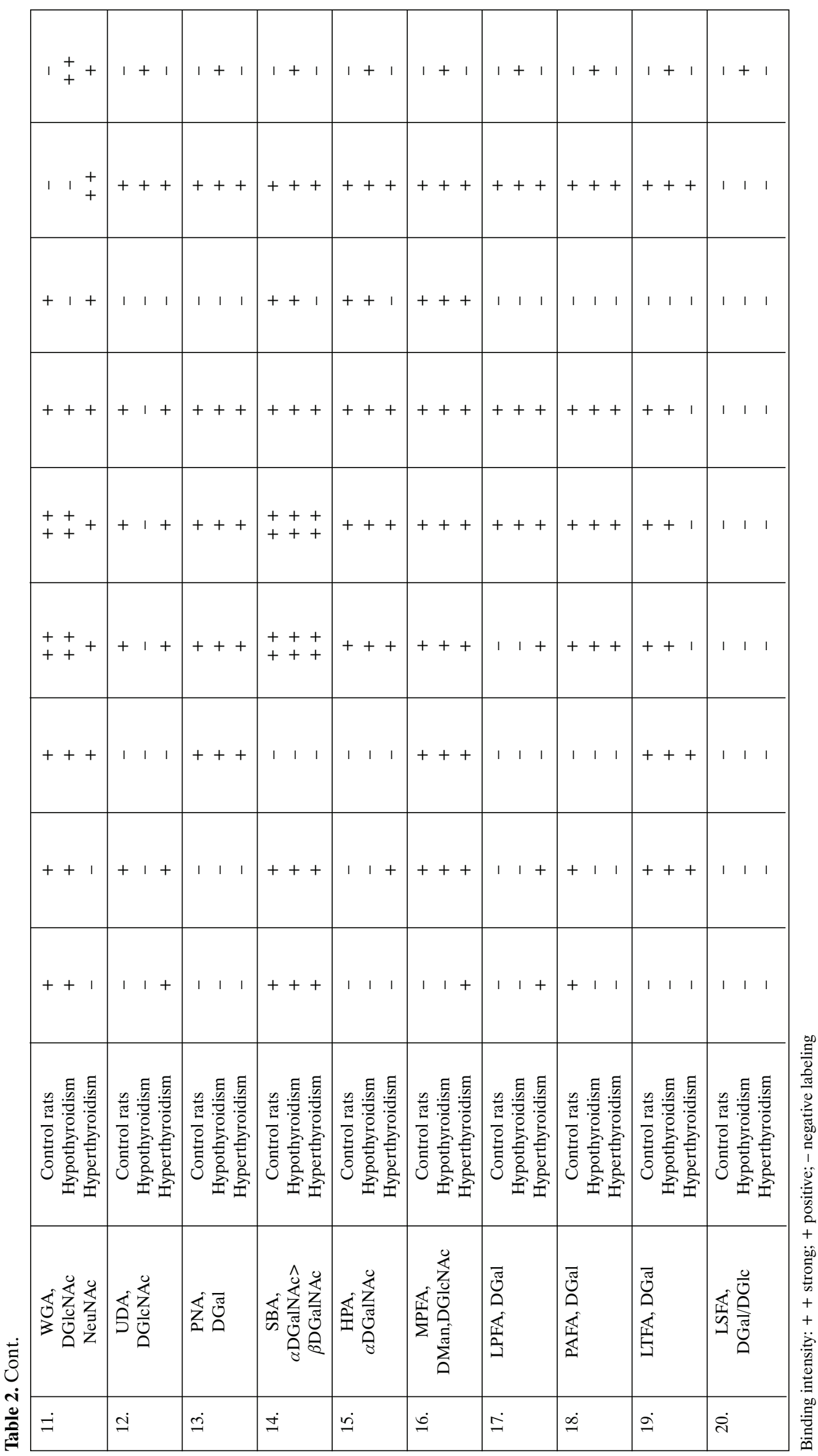


Table 3. Effects of hypothyroidism and hyperthyroidism on lectin labeling in rat endometrium

\begin{tabular}{|c|c|c|c|c|c|c|c|c|}
\hline \multirow[t]{3}{*}{$\mathbf{N}^{\circ}$} & \multirow{3}{*}{$\begin{array}{l}\text { Lectin, and its } \\
\text { carbohydrate } \\
\text { specificity }\end{array}$} & \multirow{3}{*}{$\begin{array}{l}\text { Experimental } \\
\text { group }\end{array}$} & \multicolumn{6}{|c|}{ Endometrial structures } \\
\hline & & & \multirow[b]{2}{*}{$\begin{array}{c}\text { Surface } \\
\text { epithelium }\end{array}$} & \multicolumn{2}{|c|}{ Glands } & \multirow[b]{2}{*}{$\begin{array}{l}\text { Collagen } \\
\text { fibers }\end{array}$} & \multirow[b]{2}{*}{$\begin{array}{l}\text { Pre-decidual } \\
\text { cells }\end{array}$} & \multirow[b]{2}{*}{ Leucocytes } \\
\hline & & & & Epithelium & $\begin{array}{l}\text { Secretory } \\
\text { products }\end{array}$ & & & \\
\hline 1. & $\begin{array}{c}\text { Con A, } \\
\alpha \text { DMan } / \alpha \text { DGlc }\end{array}$ & $\begin{array}{l}\text { Control rats } \\
\text { Hypothyroidism } \\
\text { Hyperthyroidism }\end{array}$ & $\begin{array}{l}++ \\
++ \\
++\end{array}$ & $\begin{array}{l}+ \\
+ \\
+\end{array}$ & $\begin{array}{l}- \\
- \\
+\end{array}$ & $\begin{array}{l}+ \\
+ \\
++\end{array}$ & $\begin{array}{l}- \\
\overline{+} \\
+\end{array}$ & $\begin{array}{c}+ \\
++ \\
-\end{array}$ \\
\hline 2. & $\begin{array}{c}\text { LCA, } \\
\alpha \text { DMan } / \alpha \text { DGlc }\end{array}$ & $\begin{array}{c}\text { Control rats } \\
\text { Hypothyroidism } \\
\text { Hyperthyroidism }\end{array}$ & $\begin{array}{l}+ \\
+ \\
+\end{array}$ & $\begin{array}{l}+ \\
+ \\
+\end{array}$ & $\begin{array}{l}- \\
- \\
-\end{array}$ & $\begin{array}{l}+ \\
+ \\
+\end{array}$ & $\begin{array}{l}+ \\
+ \\
+\end{array}$ & $\begin{array}{l}+ \\
+ \\
+\end{array}$ \\
\hline 1. & $\begin{array}{c}\text { NPA, } \\
\alpha \text { DMan }\end{array}$ & $\begin{array}{c}\text { Control rats } \\
\text { Hypothyroidism } \\
\text { Hyperthyroidism }\end{array}$ & $\begin{array}{l}+ \\
++ \\
++\end{array}$ & $\begin{array}{l}+ \\
++ \\
++\end{array}$ & $\begin{array}{l}- \\
+ \\
+\end{array}$ & $\begin{array}{c}+ \\
+ \\
++\end{array}$ & $\begin{array}{c}+ \\
+ \\
++\end{array}$ & $\begin{array}{l}++ \\
++ \\
++\end{array}$ \\
\hline 4. & $\begin{array}{l}\text { PFA, } \\
\alpha \text { LFuc }\end{array}$ & $\begin{array}{c}\text { Control rats } \\
\text { Hypothyroidism } \\
\text { Hyperthyroidism }\end{array}$ & $\begin{array}{l}+ \\
+ \\
+\end{array}$ & $\begin{array}{l}+ \\
+ \\
+\end{array}$ & $\begin{array}{l}- \\
+ \\
+\end{array}$ & $\begin{array}{l}- \\
+ \\
+\end{array}$ & $\begin{array}{l}- \\
+ \\
+\end{array}$ & $\begin{array}{l}++ \\
++ \\
++\end{array}$ \\
\hline 5. & $\begin{array}{l}\text { LLA, } \\
\alpha \text { LFuc }\end{array}$ & $\begin{array}{c}\text { Control rats } \\
\text { Hypothyroidism } \\
\text { Hyperthyroidism }\end{array}$ & $\begin{array}{l}+ \\
++ \\
++\end{array}$ & $\begin{array}{l}+ \\
++ \\
++\end{array}$ & $\begin{array}{l}+ \\
+ \\
+\end{array}$ & $\begin{array}{l}- \\
- \\
+\end{array}$ & $\begin{array}{l}- \\
- \\
+\end{array}$ & $\begin{array}{l}++ \\
++ \\
++\end{array}$ \\
\hline 6. & $\begin{array}{l}\text { LABA, } \\
\text { LFuc }\end{array}$ & $\begin{array}{c}\text { Control rats } \\
\text { Hypothyroidism } \\
\text { Hyperthyroidism }\end{array}$ & $\begin{array}{l}+ \\
++ \\
++\end{array}$ & $\begin{array}{l}+ \\
+ \\
+\end{array}$ & $\begin{array}{l}- \\
+ \\
+\end{array}$ & $\begin{array}{c}+ \\
- \\
++\end{array}$ & $\begin{array}{c}+ \\
+ \\
++\end{array}$ & $\begin{array}{l}+ \\
++ \\
++\end{array}$ \\
\hline 7. & $\begin{array}{c}\text { LASA, } \\
\text { LFuc,DGal }\end{array}$ & $\begin{array}{c}\text { Control rats } \\
\text { Hypothyroidism } \\
\text { Hyperthyroidism }\end{array}$ & $\begin{array}{c}+ \\
+ \\
+\end{array}$ & $\begin{array}{c}+ \\
+ \\
++\end{array}$ & $\begin{array}{l}+ \\
+ \\
+\end{array}$ & $\begin{array}{c}+ \\
+ \\
++\end{array}$ & $\begin{array}{c}+ \\
+ \\
++\end{array}$ & $\begin{array}{c}+ \\
++ \\
+\end{array}$ \\
\hline 8. & $\begin{array}{c}\text { EEA, } \\
\text { LFuc, } \\
\text { DGlcNAc }\end{array}$ & $\begin{array}{c}\text { Control rats } \\
\text { Hypothyroidism } \\
\text { Hyperthyroidism }\end{array}$ & $\begin{array}{l}+ \\
+ \\
+\end{array}$ & $\begin{array}{l}+ \\
+ \\
+\end{array}$ & $\begin{array}{l}- \\
- \\
-\end{array}$ & $\begin{array}{c}+ \\
- \\
++\end{array}$ & $\begin{array}{c}+ \\
+ \\
+\end{array}$ & $\begin{array}{l}+ \\
++ \\
++\end{array}$ \\
\hline 9. & $\begin{array}{c}\text { SNA, } \\
\text { NeuNAc( }(\alpha 2-6) \text { DGal }\end{array}$ & $\begin{array}{c}\text { Control rats } \\
\text { Hypothyroidism } \\
\text { Hyperthyroidism }\end{array}$ & $\begin{array}{l}++ \\
++ \\
++\end{array}$ & $\begin{array}{c}+ \\
++ \\
+\end{array}$ & $\begin{array}{l}- \\
- \\
-\end{array}$ & $\begin{array}{c}+ \\
- \\
++\end{array}$ & $\begin{array}{l}+ \\
+ \\
+\end{array}$ & $\begin{array}{l}++ \\
++ \\
++\end{array}$ \\
\hline 10. & $\begin{array}{c}\text { RCA, } \\
\beta \text { DGal } \\
\text { NeuNAc }\end{array}$ & $\begin{array}{c}\text { Control rats } \\
\text { Hypothyroidism } \\
\text { Hyperthyroidism }\end{array}$ & $\begin{array}{l}++ \\
++ \\
++\end{array}$ & $\begin{array}{c}+ \\
+ \\
++\end{array}$ & $\begin{array}{l}- \\
- \\
+\end{array}$ & $\begin{array}{l}++ \\
++ \\
++\end{array}$ & $\begin{array}{l}- \\
- \\
+\end{array}$ & $\begin{array}{c}++ \\
++ \\
-\end{array}$ \\
\hline 11. & $\begin{array}{c}\text { WGA, } \\
\text { DGlcNAc } \\
\text { NeuNAc }\end{array}$ & $\begin{array}{c}\text { Control rats } \\
\text { Hypothyroidism } \\
\text { Hyperthyroidism }\end{array}$ & $\begin{array}{l}++ \\
- \\
++\end{array}$ & $\begin{array}{l}- \\
+ \\
-\end{array}$ & $\begin{array}{l}- \\
- \\
-\end{array}$ & $\begin{array}{c}+ \\
+ \\
++\end{array}$ & $\begin{array}{l}+ \\
++ \\
++\end{array}$ & $\begin{array}{l}++ \\
++ \\
++\end{array}$ \\
\hline 12. & $\begin{array}{c}\text { UDA, } \\
\text { DGlcNAc }\end{array}$ & $\begin{array}{l}\text { Control rats } \\
\text { Hypothyroidism } \\
\text { Hyperthyroidism }\end{array}$ & $\begin{array}{l}+ \\
+ \\
+\end{array}$ & $\begin{array}{l}+ \\
+ \\
+\end{array}$ & $\begin{array}{l}- \\
- \\
+\end{array}$ & $\begin{array}{c}+ \\
+ \\
++\end{array}$ & $\begin{array}{c}+ \\
+ \\
++\end{array}$ & $\begin{array}{c}- \\
++ \\
-\end{array}$ \\
\hline 13. & $\begin{array}{l}\text { PNA, } \\
\text { DGal }\end{array}$ & $\begin{array}{c}\text { Control rats } \\
\text { Hypothyroidism } \\
\text { Hyperthyroidism }\end{array}$ & $\begin{array}{l}+ \\
+ \\
+\end{array}$ & $\begin{array}{l}+ \\
+ \\
+\end{array}$ & $\begin{array}{l}- \\
- \\
-\end{array}$ & $\begin{array}{l}+ \\
+ \\
+\end{array}$ & $\begin{array}{c}+ \\
+ \\
++\end{array}$ & $\begin{array}{l}+ \\
++ \\
++\end{array}$ \\
\hline 14. & $\begin{array}{c}\text { SBA, } \\
\alpha \text { DGalNAc } \\
\beta \text { DGalNAc }\end{array}$ & $\begin{array}{c}\text { Control rats } \\
\text { Hypothyroidism } \\
\text { Hyperthyroidism }\end{array}$ & $\begin{array}{l}+ \\
++ \\
++\end{array}$ & $\begin{array}{l}+ \\
++ \\
++\end{array}$ & $\begin{array}{c}+ \\
+ \\
++\end{array}$ & $\begin{array}{c}+ \\
+ \\
++\end{array}$ & $\begin{array}{c}+ \\
+ \\
++\end{array}$ & $\begin{array}{c}+ \\
++ \\
+\end{array}$ \\
\hline 15. & $\begin{array}{c}\text { HPA, } \\
\alpha \text { DGalNAc }\end{array}$ & $\begin{array}{c}\text { Control rats } \\
\text { Hypothyroidism } \\
\text { Hyperthyroidism }\end{array}$ & $\begin{array}{l}+ \\
+ \\
+\end{array}$ & $\begin{array}{l}+ \\
+ \\
+\end{array}$ & $\begin{array}{l} \\
- \\
-\end{array}$ & $\begin{array}{l}+ \\
+ \\
+\end{array}$ & $\begin{array}{l}+ \\
+ \\
+\end{array}$ & $\begin{array}{l}+ \\
++ \\
++\end{array}$ \\
\hline 16. & $\begin{array}{c}\text { MPFA, } \\
\text { DMan, } \\
\text { DGlcNAc }\end{array}$ & $\begin{array}{c}\text { Control rats } \\
\text { Hypothyroidism } \\
\text { Hyperthyroidism }\end{array}$ & $\begin{array}{l}++ \\
++ \\
++\end{array}$ & $\begin{array}{l}+ \\
+ \\
+\end{array}$ & $\begin{array}{l}+ \\
+ \\
+\end{array}$ & $\begin{array}{c}+ \\
+ \\
++\end{array}$ & $\begin{array}{c}+ \\
+ \\
++\end{array}$ & $\begin{array}{c}- \\
++ \\
+\end{array}$ \\
\hline 17. & $\begin{array}{l}\text { LPFA, } \\
\text { DGal }\end{array}$ & $\begin{array}{c}\text { Control rats } \\
\text { Hypothyroidism } \\
\text { Hyperthyroidism }\end{array}$ & $\begin{array}{l}+ \\
- \\
-\end{array}$ & $\begin{array}{l}+ \\
- \\
-\end{array}$ & $\begin{array}{l}- \\
- \\
-\end{array}$ & $\begin{array}{c}+ \\
+ \\
++\end{array}$ & $\begin{array}{c}+ \\
+ \\
++\end{array}$ & $\begin{array}{l}+ \\
+ \\
+\end{array}$ \\
\hline 18. & $\begin{array}{l}\text { PAFA, } \\
\text { DGal }\end{array}$ & $\begin{array}{c}\text { Control rats } \\
\text { Hypothyroidism } \\
\text { Hyperthyroidism }\end{array}$ & $\begin{array}{c}+ \\
+ \\
++\end{array}$ & $\begin{array}{c}+ \\
+ \\
++\end{array}$ & $\begin{array}{l}- \\
- \\
+\end{array}$ & $\begin{array}{c}+ \\
+ \\
++\end{array}$ & $\begin{array}{c}+ \\
+ \\
++\end{array}$ & $\begin{array}{l}++ \\
++ \\
++\end{array}$ \\
\hline 19. & $\begin{array}{l}\text { LTFA, } \\
\text { DGal }\end{array}$ & $\begin{array}{c}\text { Control rats } \\
\text { Hypothyroidism } \\
\text { Hyperthyroidism }\end{array}$ & $\begin{array}{l}- \\
- \\
-\end{array}$ & $\begin{array}{l}- \\
- \\
-\end{array}$ & $\begin{array}{l}- \\
- \\
-\end{array}$ & $\begin{array}{c}+ \\
+ \\
++\end{array}$ & $\begin{array}{l}+ \\
+ \\
+\end{array}$ & $\begin{array}{l}+ \\
+ \\
+\end{array}$ \\
\hline 20. & $\begin{array}{c}\text { LSFA, } \\
\text { DGal/DGlc }\end{array}$ & $\begin{array}{c}\text { Control rats } \\
\text { Hypothyroidism } \\
\text { Hyperthyroidism }\end{array}$ & $\begin{array}{l}- \\
- \\
-\end{array}$ & $\begin{array}{l}- \\
- \\
-\end{array}$ & $\begin{array}{l}- \\
- \\
-\end{array}$ & $\begin{array}{l}- \\
- \\
-\end{array}$ & $\begin{array}{l}- \\
- \\
-\end{array}$ & $\begin{array}{l}- \\
- \\
-\end{array}$ \\
\hline
\end{tabular}

Binding intensity: + + strong; + positive; - negative labeling 
Both hyper- and hypothyroidism induced significant redistribution of DMan, LFuc, NeuNAc, DGlcNAc, DGalNAc, and DGal determinants in the ovaries and endometrium. The most representative results are summarized in Tables 2-3 and Figures 1-3. It is noteworthy that the reduction of certain residues was combined with overexposure of others, but different structural elements of the ovaries and endometrium demonstrated rather specific modes of such redistribution.

In particular, hypothyroidism induced enhanced SBA reactivity of ovarian follicles granulosa cells and cytoplasmic granularity of oocytes (Figure 1). Collagen fibers in the endometrium of hyperthyroid rats expressed additional receptor sites for LABA, SNA, MPFA, LPFA, LTFA, and PAFA, while in control rats, these fibers were only slightly positive with LABA, LPFA, MPFA and SNA (Figure 2). Selective lectin labeling of leucocytes was useful for demonstrating hypothyroidism-induced accumulation of these cells within ovarian and endometrial stroma, even in cases when the surrounding tissues expressed reduced lectin reactivity (Figure 3 ). It was also shown that hyperthyroidism altered ovarian and endometrial glycoconjugates more strongly than hypothyroidism; the endometrium was more susceptible to thyroxine-modulated impairments than the ovaries.

Rats given mercazolil with $30 \mu \mathrm{g} / \mathrm{kg}$ of thyroxin demonstrated normal leucocyte counts and normal amounts of collagen fibers, as well as normal lectinbinding characteristics in the ovaries and endometrium, except for slightly reduced reactivity of collagen fibers with SBA, HPA, and PNA. Mercazolil given in combination with $1.6 \mu \mathrm{g} / \mathrm{kg}$ of thyroxin was accompanied by all of the typical signs of induced hypothyroidism, thus proving that the above dose provided an insufficient replacement.

\section{Discussion}

The present observations of the morphological and functional changes in the ovaries and endometrium correlate with previously reported data $[16,17]$ and indicate the negative effects of both hyper- and hypothyroidism on the female reproductive system. It is most likely that an altered balance of thyroid hormones affects the hypothalamic-pituitary-gonadal axis $[5,7]$. Disorders in the hormonal activity of the ovaries apparently induce the histopathological changes in the endometrium.

The present data on increased folliculogenesis and inhibited corpora lutea formation under hypothyroid influence are to a certain extent at variance with the results reported by Hapon et al. [18], who described prolonged activity of corpus luteum gravidarum in hypothyroid rats. At the same time, the present results are in good agreement with the clinical observations of polycystic ovary syndrome associated with hypothyroidism [19].

The present findings on decreased lectin reactivity of collagen fibers in hypothyroid endometrium support the earlier observations of Saha et al. [20], who reported decreased synthesis in combination with enhanced degradation of collagen fibers in hypothyroid rat ovaries. We detected an adverse reaction - extra collagenization of hyperthyroid endometrium, probably resulting from the stimulatory influence of hyperthyroidism on all cells, including fibroblasts, thus leading to the increased production of collagen fibers. Moreover, thyroxin stimulates production of progesterone releasing factor [7], which, via the effect of progesterone, apparently stimulates the accumulation of pre-decidual cells.

Our results on the intense lectin binding to ovarian and endometrial constituents confirm the observations of others on the vast diversity and important role of glycoconjugates in the histophysiology of the female reproductive system $[9,12]$. The diversity of different classes of carbohydrates in the ovaries (Figure 4) and endometrium apparently results in multiple possible forms of rearrangement under experimental conditions.

It can be speculated that, induced by hyper- and hypothyroidism, the reported trends in the redistribution of lectin receptor sites could involve two main mechanisms: (1) incomplete final glycosylation patterns of tissue carbohydrates, resulting in exposure of subterminal sugar residues; (2) alterations of lysosomal exoglycosidases (i.e. D-mannosidase, L-fucosidase, D-galactosidase, $\mathrm{N}$-acetyl-D-hexosaminidase) synthesis, leading to the accumulation or unmasking of certain lectin receptor sites (Figure 4). The latter mechanism is supported by the recent observations of Szajda et al. [21], who reported exoglycosidases impairments in patients with neoplasia.

\section{Conclusions}

1. Thyroid disorders induce significant redistribution of carbohydrate determinants in female reproductive organs: hyperthyroidism causes more severe alterations of glycoconjugates than hypothyroidism, with the endometrium being more susceptible than the ovaries.

2. New lectin preparations from fungi (MPFA, LPFA, LTFA, PAFA, LSFA) proved to be useful tools for selective labeling of ovarian and endometrial constituents.

3. Lectin histochemistry methods can be recommended for monitoring the efficacy of pharmacological treatment of endocrine pathologies. 

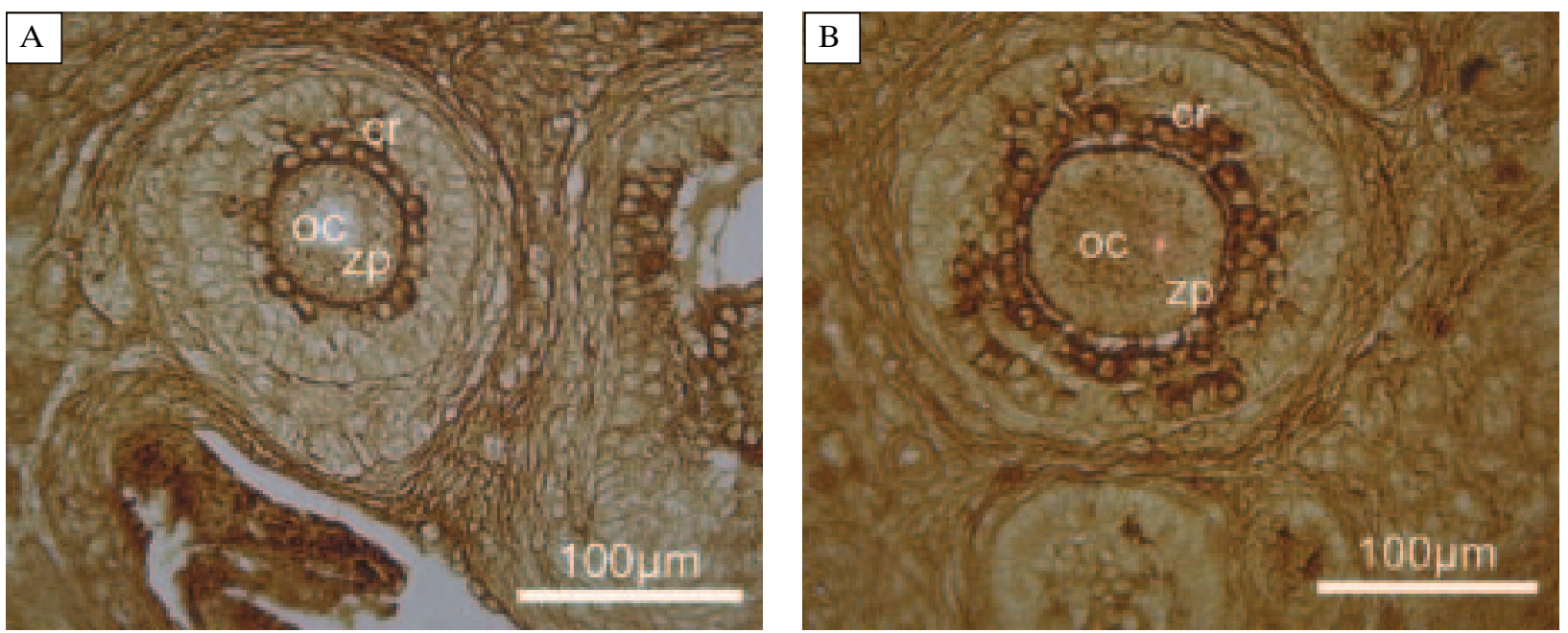

Figure 1. SBA labeling in growing ovarian follicles of control (A) and hypothyroid (B) rats. Enhanced reactivity of granulosa cells and cytoplasmic granularity of oocytes induced by hypothyroidism are seen: oc — oocyte; zp — zona pellucida; cr - corona radiata. Original magnification $\times 400$
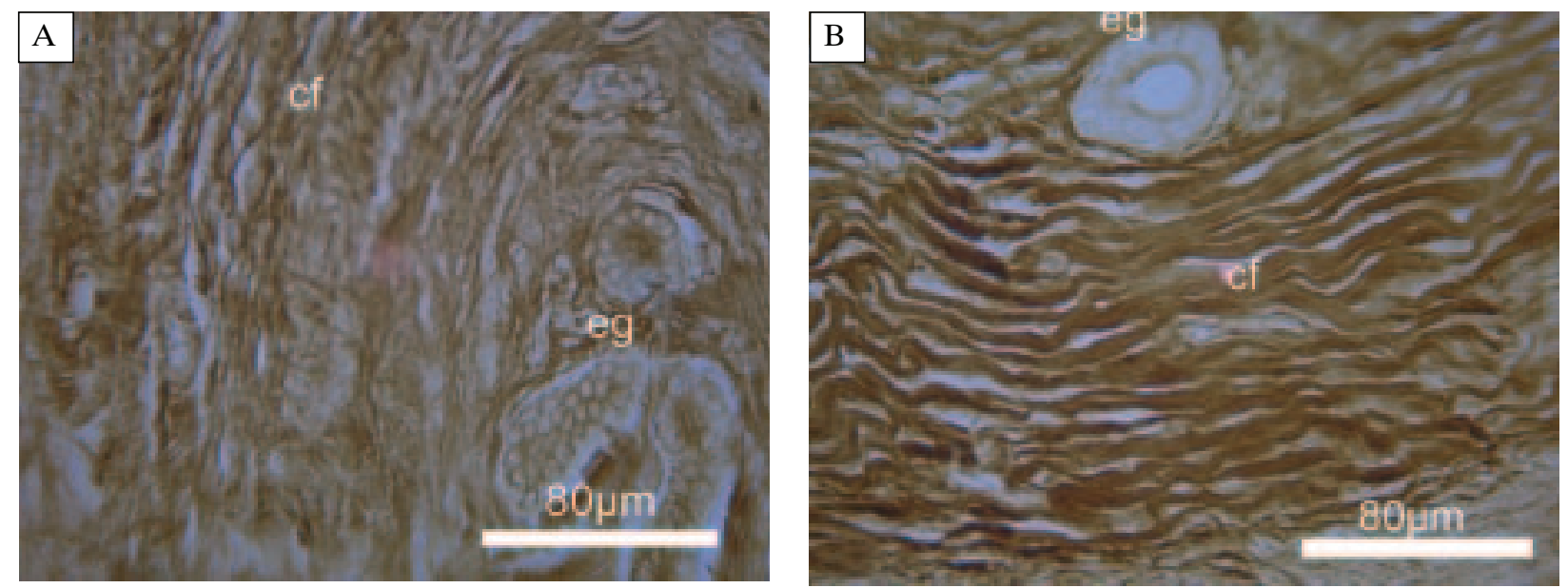

Figure 2. Micromorphology of control (A) and hyperthyroid (B) rat endometrium. Thick bundles of collagen fibers show increased lectin reactivity (B) with the MPFA lectin label: eg — endometrial gland; cf — collagen fibers. Original magnification $\times 400$

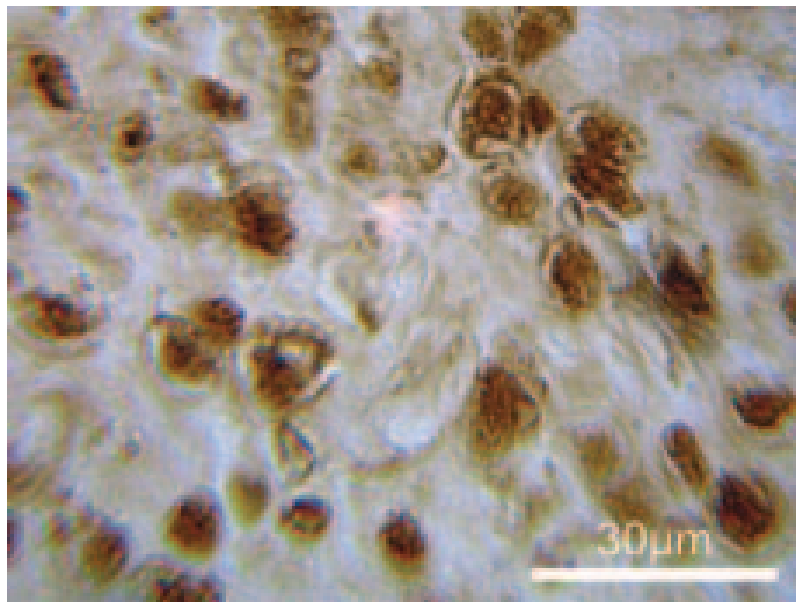

Figure 3. Infiltration of hypothyroid rat endometrium with leucocytes as revealed by HPA lectin binding.

Original magnification $\times 900$

\section{Acknowledgements}

The authors are very grateful to D. Pharm. Sci. V. Antonyuk, for providing the lectin-peroxidase kits and to Professor A. Sogomonian for reading and commenting on the manuscript. The authors would also like to thank Mrs. Svitlana Riznychok for technical help, and Dr. Luba Wolchuk and Dr. Anthony Shardt for their editorial advice and assistance during the preparation of this paper.

\section{References}

1. Siraj ES. Update on the diagnosis and treatment of hyperthyroidism. J Clin Outc Manag. 2008;15:298-307.

2. Muravliova OV, Riabushko NN, Bobyrieva LE. Prevalence of thyroid pathology in Poltava region in post-Chornobyl era and ways for its prevention. Odessa Med J (Odessa). 2004;5:96-98. 


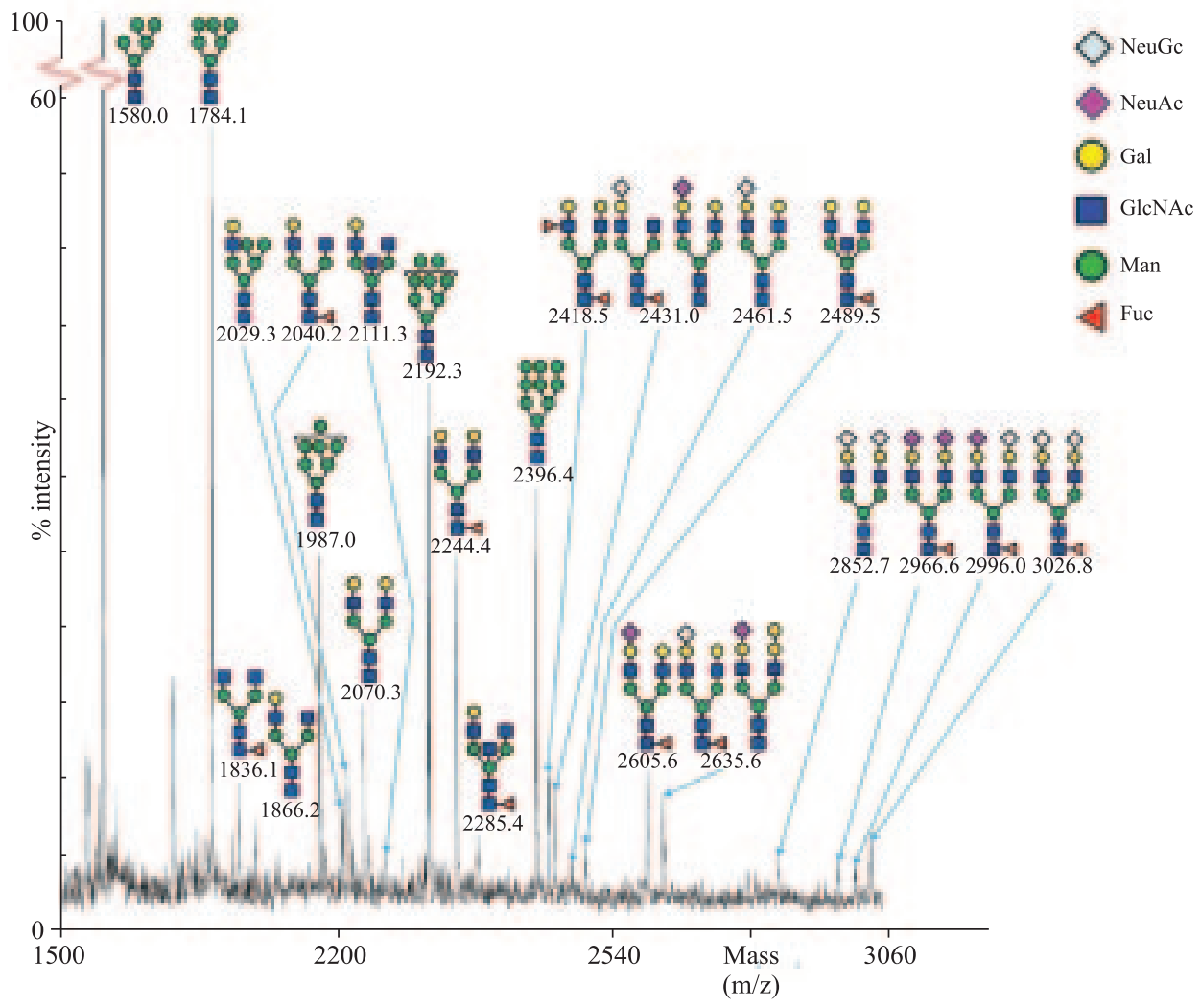

Figure 4. The main types of N-glycans found within the ovarian tissue of C57BL/6 mice on mass spectrometry, according to the Consortium for Functional Glycomics (http://functionalglycomics.org)

3. Pankiv VI. Prevalence of thyroid pathology in iodine deficient regions of western Ukraine. Endocrinology (Kyiv). 2006;11:134-137.

4. Perelman AH, Clemons RD. The fetus in maternal hyperthyroidism. Thyroid. 1992;2:225-228.

5. Abe Y, Momotani N. Thyroid disease and reproduction dysfunction. Nippon Rinsho. 1997;55:2974-2978.

6. Inzucchi SE, Burrow GN. The thyroid gland and reproduction. In: Reproductive Endocrinology. Philadelphia, Saunders, 1999:413-435.

7. Doufas AG, Mastorakos G. The hypothalamic-pituitarythyroid axis and the female reproductive system. Ann NY Acad Sci. 2000;900:65-76.

8. Johnson CA. Thyroid issues in reproduction. Clin Tech Small Pract. 2002;17:129-132.

9. Skutelsky E, Ranen E, Shalgi R. Variations in the distribution of sugar residues in the zona pellucida as possible species-specific determinants of mammalian oocytes. JReprod Fertil. 1994;100:35-41.

10. Shalgi R, Raz T. The role of carbohydrate residues in mammalian fertilization. Histol Histopath. 1997;12:813-822.

11. Miranda PV, Gonzales-Echeverria F, Blaquier JA et al. Evidence for the participation of beta hexaminidase in human sperm-zona pellucida interaction in vitro. Mol Hum Reprod. 2000;6:699-706.

12. Jimenes-Movilla M, Aviles M, Gomes-Torres MJ et al. Carbohydrate analysis of the zona pellucida and cortical granules of human oocytes by means of ultrastructural cytochemistry. Hum Reprod. 2004;19:1842-1855.

13. Alberts B, Johnson A, Lewis J et al. Molecular biology of the cell, $5^{\text {th }}$ ed. New York, Garland; 2008:1257-1305.
14. Antonyuk VO, Yastchenko AM, Antonyuk RV, Ambarova NO. Carbohydrate specificity of lectin, purified from the fruiting bodies of Mycena pura /Fr./Kumm. and its use in histochemical investigation. Biopolym Cell (Kyiv). 2009;25:466-475.

15. Brooks SA, Leathem AJC, Shumacher U. Lectin histochemistry: a concise practical handbook. Oxford; BIOS: 1997.

16. Jiang JY, Miyabayashi K, Nottola SA et al. Thyroxine treatment stimulated ovarian follicular angiogenesis in immature hypothyroid rats. Histol Histopathol. 2008;23:1387-1398.

17. Hatsuta M, Abe K, Tamura K et al. Effect of hypothyroidism on the estrous cycle and reproductive hormones in mature female rats. Eur J Pharmacol. 2004;486:343-348.

18. Hapon MB, Motta AB, Ezquer M et al. Hypothyroidism prolongs corpus luteum function in the pregnant rat. Reproduction. 2007;133:197-205.

19. Panico A, Lupoli GA, Fonderico F et al. Multiple ovarian cysts in a young girl with severe hypothyroidism. Thyroid. 2007;17:1289-1293.

20. Saha SK, Ghosh P, Konar A et al. Differential expression of procollagen lysine 2-oxoglutarase and matrix metalloproteinase isoforms in hypothyroid rat ovary and disintegration of extracellular matrix. Endocrinology. 2005;146:2963-2975.

21. Szajda SD, Waszkiewicz N, Stypulkowska, A et al. Lysosomal exoglycosidases in serum and urine of patients with pancreatic adenocarcinoma. Folia Histochem Cytobiol. 2010;48:351-357.

Submitted: 4 July, 2011

Accepted after reviews: 24 April, 2012 